\title{
ON SCALE INVARIANCE AND ANOMALIES IN QUANTUM MECHANICS*
}

\author{
A. Cabo ${ }^{\dagger}$, J.L. Lucio and H. Mercado \\ Instituto de Física, Universidad de Guanajuato \\ Apartado Postal E-143, León, Gto., México
}

\begin{abstract}
We re-consider the quantum mechanics of scale invariant potentials in two dimensions. The breaking of scale invariance by quantum effects is analyzed by the explicit evaluation of the phase shift and the self-adjoint extension method. We argue that the breaking of scale invariance reported in the literature for the $\delta(\mathbf{r})$ potential, is an example of explicit and not an anomaly or quantum mechanical symmetry breaking.
\end{abstract}

*Work supported by CONACyT under contract 39798-E

${ }^{\dagger}$ On leave of absence Instituto de Cibernética, Matemáticas y Física, Calle E No. 309 Esq. 15 Vedado, La Habana, Cuba 


\section{INTRODUCCION}

Symmetries play a central role in the description of physical systems. Well known examples [1] are the space-time symmetries (space and time homogeneity, i.e. invariance of the system under space and time translations) which are the basis for the energy and momentum conservation laws we learn to use in elementary classical physics courses. The link between symmetry properties of a system and conservation laws is provided by Noether's theorem [2] which asserts that associated to each transformation that leaves invariant the action there exist a conserved quantity. The properties of the system can, in general, be obtained in terms of such conserved quantities without completely solving the equations of motion that describe the system. Besides the space-time symmetries there may exist "internal" symmetries which are related to the conservation of quantities such as the electric charge. In fact, symmetry requirements are enough to fix the way electric charges interact, thus the question of what are the interactions that ocurr in nature is traded by the more fundamental question: what are the symmetries of nature?

In the conventional approach, theories are formulated at the classical level and latter quantized according to a well established procedure [3]. Usually the symmetries survive the process of quantization ensuring thus the validity of conservation laws at the quantum level. Remember also that although the electromagnetic and the gravitational are long range interactions which may be deal with at the classical level, the weak and strong are very short range interactions and inevitably require a quantum treatment. If we want to describe the interactions in terms of symmetries, we have to make sure that the symmetry is valid at the quantum level. Anomalies occur when the symmetry is destroyed by quantization, a phenomenon originally identified in quantum field theory and recently analyzed in the context of quantum mechanics [4]. In quantum field theory anomalies may have phenomenological consequences (when the anomaly is associated to a global symmetry) or render inconsistent the theory (if the anomaly is related to a local symmetry), this has motivated the interest in anomaly free theories and the search for a deeper understanding of this symmetry breaking mechanism. Anomalies have not raised much interest in the framework of quantum mechanics, in fact only a few examples [5] have been analyzed in detail, although the possibility that they are related to geometrical phases has been advanced in this context [6].

Systems invariant under dilation of the space-time coordinates $\left(x \rightarrow \rho^{-1 / 2} x\right.$ with $\rho$ an arbitrary dimensionles parameter) are refereed as scale invariant, a characteristic of systems not depending on dimensional parameters. In quantum field theory this symmetry is destroyed by quantum effects and a similar conclusion has been obtained in quantum mechanics [7]. In nonrelativistic physics, where the kinetic part of the Hamiltonian is proportional to $\mathbf{p}^{2}$, scale invariant systems are described by potentials such that $V\left(\rho^{-1 / 2} \mathbf{r}\right)=$ $\rho V(r)$ (we say the potential and the Hamiltonian scale as $\rho$ ). The invariance of the system under this transformation follows from the fact that modifications of the Hamiltonian by an overall factor does not affect the equation of motion. Examples of mechanical scale invariant systems are the $1 / r^{2}$ and, in two dimensions, the delta potential $\delta^{2}(\mathbf{r})$. Scattering off the $1 / r^{2}$ potential is exactly solvable, it yields an energy independent phase shift which has to be understood as a signal of scale invariance at the quantum level, i.e. for this system the 
symmetry survives quantization. This is a clean example where the methods to be used in more involved analysis can be tested.

The interest in the delta potential arose from the study of the $\lambda \varphi^{4}$ theory, which is non trivial perturbatively, but suspected to be non interacting. Beg and Furlong [8] considered the non-relativistic limit of the $\lambda \varphi^{4}$ —which results in the quantum mechanics of the delta potential - in order to get some insight into the behavior of the full theory. They concluded that for a finite, unrenormalized coupling constant, a trivial S matrix is obtained. Notice that we advocated renormalization, a subtraction procedure required in quantum field theory where singularities associated to short distances (ultraviolet divergences) have to be removed by renormalizing the Lagrangian, which amounts to redefine the fields, the coupling and the mass. The strong singularity of the delta potential, and its "contact" nature, suggest that regularization is required in order to properly define the quantum mechanics of that system. In three dimensions, and also in two [9], it is possible to get non-trivial dynamics at the price of renormalizing the interaction. The two dimensional case has the further interest of scale invariance at the classical level and the possibility it offers to study the survival of this symmetry to the quantization process. For that reason the two dimensional delta potential has been considered a pedagogical laboratory where field theoretical concepts such as renormalization [10], renormalization group equation [11], anomalies [4,7] and dimensional transmutation [12] can be studied.

The regularization procedure used to treat singular potential in quantum mechanics must posses several features; in particular it should preserve the symmetries, otherwise the results obtained for physical observable are meaningless as they do not reflect the properties of the original system. It is possible to argue that the regularization and renormalization are part of the quantization procedure, however the breaking of scale invariance is not an intrinsic characteristic of the regularization of the delta potential; it is indeed possible to envisage a symmetry preserving regularization procedure. This point has been overlooked in previous analysis, which define the potential in terms of a distribution sharing some properties of Dirac's delta, but not scale invariance [10]. In this paper we introduce a distribution with the adequate scaling properties and explicitly work out the scattering problem to evaluate the phase shift (using Green functions and solving exactly the corresponding Schrödinguer Equation). The distinctive feature of this approach is that it requires regularization but not renormalization leading thus, in agreement with Beg and Furlong [8] and Jackiw [9], to a trivial S matrix. Thus, we conclude that the breaking of scale invariance reported in the literature is not an anomaly but an explicit breaking.

In classical mechanics the Poisson bracket of Noether's charge with a dynamical variable yields the variation of such a variable under the symmetry transformation, for that reason the charge is called the symmetry generator. In quantum mechanics the Poisson bracket is replaced by the commutator, with the further constraint that the generator must be Hermitean (which may require solving ordering ambiguities to avoid inconsistencies). This quantum mechanical charge is then used to build the unitary operator which carries the corresponding transformation in Hilbert space. Cocycles are phases which appear as a necessary generalization of the group representation theory in quantum mechanics when the 
action, but not the lagrangian, is invariant under a symmetry transformation [13]. Usually one is not faced with cocycles because they are trivial, i.e. it is possible to redefine the wave function and the operators to avoid them. If the cocyle appears and it is non trivial, this signals a symmetry breaking by quantum effects. Originally our interest in the delta potential was to show that cocycles is an alternative framework to analyze the breaking of scale invariance by quantum effects. We have been unable to reach our goal due to complications arising from the non-point nature of the scale transformations.

An alternative way to test for a symmetry after quantization is the self-adjoint extension. In the particular case of scale invariance and the delta potential, the method relies on the behavior around the origin of the wave function and the fact that this must be invariant under a symmetry transformation, i.e. the relation between $\psi(0)$ and $\psi^{\prime}(0)$ can not change by the action of the symmetry generator. This behavior at the origin is the bridge between the self-adjoint property of the Hamiltonian and the symmetry properties of the system. The reason is that the Hamiltonian for a free particle in two dimensions is not self-adjoint and that means that only certain class of functions -those having at the origin derivative proportional to the function it self- are acceptable as solutions for this problem [16]. Different self-adjoint extensions correspond to different relations $\psi(0)=\lambda \psi^{\prime}(0)(\lambda$ is called the selfadjoint extension parameter) and $\lambda$ is related to the phase shift, therefore different $\lambda^{\prime} s$ correspond to different potentials. In the main text we discuss in detail the self-adjoint extension for the $\delta(\mathbf{r})$ potential. We show that the result of this analysis is completely consistent with that obtained by the explicit evaluation of the phase shift. We remark that this approach provides information about the symmetries of the system at the quantum level, but not on the nature of the symmetry breaking mechanism, in particular this is not a criteria for the existence of anomalies.

Summarizing, at the classical level the two dimensional delta potential defines a system for which the action (but not the Lagrangian, which implies the appearance of cocycles) is invariant under scale transformations. For the same system in quantum mechanics we face the following alternative: a) we deal with a finite, unrenormalized coupling constant, a trivial $\mathrm{S}$ matrix and clearly scale invariance $b$ ) after renormalization of the coupling constant, the delta potential leads to a non trivial S matrix and an energy dependent phase shift indicating the breaking of scale invariance. Besides the explicit calculation of the energy dependent phase shift, Jackiw confirmed [9] these results in terms of the self adjoint extension. Thus the breaking of scale invariance is out of the question, however and that is the point we address in this paper, the nature of the breaking is not evident. In fact, and contrary to some claims in the literature, we argue that this is an example of explicit and not an anomaly or quantum mechanical symmetry breaking.

\section{SCALE INVARIANCE.}

In the following we restrict our attention to two dimensions. The vector components are labeled by Latin indices $i=1,2$. The finite scale transformation are defined by:

$$
\begin{aligned}
t \stackrel{T}{\longrightarrow} t^{\prime}=\rho t & x_{i}(t) \stackrel{T}{\longrightarrow} x_{i}^{\prime}\left(t^{\prime}\right)=\rho^{-1 / 2} x_{i}(\rho t), \\
& p_{i}(t) \stackrel{T}{\longrightarrow} p_{i}^{\prime}\left(t^{\prime}\right)=\rho^{1 / 2} p_{i}(\rho t) .
\end{aligned}
$$


We consider the $1 / r^{2}$ and the $\delta^{2}(\mathbf{r})$ potential which have the same properties under scale transformations $\left(U(r) \stackrel{T}{\longrightarrow} U\left(\rho^{-1 / 2} r\right)=\rho U(r)\right)$ :

$$
\begin{gathered}
\frac{1}{r^{2}} \stackrel{T}{\longrightarrow} \frac{1}{r^{2}}=\frac{\rho}{r^{2}}, \\
\delta^{2}(\mathbf{r}) \stackrel{T}{\longrightarrow} \delta^{2}\left(\mathbf{r}^{\prime}\right)=\rho \delta^{2}(\mathbf{r}) .
\end{gathered}
$$

A conventional approach to regularize the $\delta(\mathbf{r})$ potential amounts to the replacement [10]:

$$
\delta^{2}(\mathbf{r}) \longrightarrow \begin{cases}\frac{v(a)}{\pi a^{2}} & r \leq a \\ 0 & r>a\end{cases}
$$

In the following we consider two possibilities for $v(a)$ :

$$
\begin{aligned}
& v(a)=\frac{2 \pi}{\ln \left(a / a_{0}\right)+\gamma}, \\
& v(a)=v=\text { constant }
\end{aligned}
$$

The first (3a), has been used in the literature [10] in order to obtain a non trivial S matrix. Below we argue that $(3 \mathrm{~b})$ leads to a more appropriated regularization of the $\delta(\mathbf{r})$ potential.

Under scale transformation the "regularized potential" transforms according to:

$$
U\left(r^{\prime}\right)= \begin{cases}\frac{v(a)}{\pi a^{2}} & r^{\prime}=\frac{r}{\sqrt{\rho}} \leq a \rightarrow r \leq \sqrt{\rho} a \equiv a^{\prime}, \\ 0 & r^{\prime}=\frac{r}{\sqrt{\rho}}>a \rightarrow r>\sqrt{\rho} a \equiv a^{\prime}\end{cases}
$$

which can be rewritten as:

$$
U\left(r^{\prime}\right)= \begin{cases}\frac{\rho v\left(a^{\prime} / \sqrt{\rho}\right)}{\pi a^{\prime 2}} & r \leq a^{\prime} \\ 0 & r>a^{\prime}\end{cases}
$$

In the $a \rightarrow 0$ limit $(2,3)$ describes a "contact" or "zero range" potential, however it does not share with the $\delta(\mathbf{r})$ the properties under scale transformations, unless $v(a)$ is $a$ independent. Therefore, by using the distribution $(2,3 \mathrm{a})$, and before doing any quantum mechanics, one introduces an explicit breaking of scale invariance. On the other hand (3b) defines a family of probe functions appropriated for a mathematical definition of the $\delta^{2}(\mathbf{r})$, and also ensures the adequate scale transformation properties of the regularized $\delta^{2}(\mathbf{r})$ potential.

Notice that for infinitesimal transformations $\left(\rho \equiv 1+\epsilon+O\left(\epsilon^{2}\right)\right)$, scaling (Eq.(1)) involve the velocities, i.e. these are non point transformations which may render difficult its implementation in quantum mechanics. Both for the $1 / r^{2}$ and the $\delta^{2}(\mathbf{r})$ potentials, the variation of the Lagrangian under the infinitesimal scale transformation is the time derivative of the Lagrangian $\delta \mathcal{L}=\frac{d \mathcal{L}}{d t}$. This non vanishing variation ensures the appearance of cocycles once the transformation is implemented at the quantum level [13]. The charge associated to this symmetry is obtained through Noether's theorem [2] 


$$
\mathbf{D}=\sum_{i} \frac{\partial \mathcal{L}}{\partial \dot{x}_{i}} \delta x_{i}-\mathcal{L}=\mathcal{H} t-\frac{1}{2} \mathbf{p} \cdot \mathbf{r} .
$$

It is straightforward to check that both at the classical and quantum level $\mathbf{D}$ generates the infinitesimal scale transformations:

$$
\begin{gathered}
\left\{x_{i}, \mathbf{D}\right\}=\dot{x}_{i} t-\frac{1}{2} x_{i}\left\{\dot{x}_{i}, \mathbf{D}\right\}=\ddot{x}_{i} t+\frac{1}{2} \dot{x}_{i}, \\
-\left[x_{i}, \mathbf{D}\right]=\dot{x}_{i} t-\frac{1}{2} x_{i}\left[\dot{x}_{i}, \mathbf{D}\right]=\ddot{x}_{i} t+\frac{1}{2} \dot{x}_{i} .
\end{gathered}
$$

\section{EXACT SOLUTIONS.}

Let us consider the scattering, in two dimensions, of a particle of mass $m$ by a central potential $U(r)$. The hamiltonian of the system is

$$
\mathcal{H}=\frac{\mathbf{p}^{2}}{2 i n}+U(r)
$$

For central potentials and in two dimensions, the angular momentum eigenfunctions $e^{i \ell \theta}$ are used to reduce the Schrödinguer equation

$$
\psi(\mathbf{r})=\varphi(r) e^{i \ell \theta},
$$

the radial wave function $\varphi(r)$ is obtained as a solution to

$$
\left(\frac{d^{2}}{d r^{2}}+\frac{1}{r} \frac{d}{d r}-\frac{\nu^{2}}{r^{2}}+\kappa^{2}\right) \varphi(r)=0
$$

where

$$
\begin{gathered}
\nu^{2}(\ell)=\ell^{2}+2 m \lambda, \quad \kappa^{2}=k^{2}=2 m E, \quad \text { if } U(r)=\lambda / r^{2} \\
\nu^{2}(\ell)=\ell^{2}, \quad \kappa_{\delta}^{2}= \begin{cases}k^{2}=2 m E, & r>a \\
\kappa^{2}=k^{2}-2 m \frac{v(a)}{\pi a^{2}}, & r \leq a, \text { if } U(r) \text { iven by }(2,3) .\end{cases}
\end{gathered}
$$

For fractional $\nu$, two independent solutions [14] are the first $J_{\nu}(\kappa r)$ and second class $Y_{\nu}(\kappa r)$ Bessel functions. For $\nu$ integer and $r<a$ two independent solutions are the modified Bessel functions $I_{\nu}(\kappa r)$ and $K_{\nu}(\kappa r)$.

\section{$1 / r^{2}$ potential.}

The $Y_{\nu}(\kappa r)$ function is discarded due to its singular behavior at the origin, therefore the physically acceptable radial wave function is given by: 


$$
\psi_{\ell}(r, \theta)=N J_{\nu(\ell)}(k r) e^{i \ell \theta}, \quad \ell=-\infty, \ldots \infty .
$$

On the other hand, the solution corresponding to an outgoing free wave has the asymptotic behavior:

$$
\psi_{\ell}^{A}(r, \theta) \rightarrow \frac{e^{i \ell \theta}}{\sqrt{r}} \cos \left(k r-\frac{\ell \pi}{2}-\frac{\pi}{4}+\delta_{\ell}\right) .
$$

The scattering phase shift is obtained by comparing the asymptotic expansions of $(6,7)$ (the coefficient $\mathrm{N}$ is chosen so that, for $U(r)=0$ the solutions coincide everywhere).

$$
\delta_{\ell}(k)=\frac{\pi}{2}\left(\ell-\sqrt{\ell^{2}+2 m \lambda}\right) .
$$

This expression shows that phase shift is energy $(k)$ independent, which is the signature for scale invariance.

One may question this derivation due to the long range behavior of the $1 / r^{2}$ potential. Further details about this problem are presented in the following section, where the phase shift is calculated using the scattering wave function.

\section{$\delta^{2}(\mathbf{r})$ potential}

Since this potential is not a function but a distribution, we consider the set of spherically symmetric potentials vanishing outside a circle of radius $a$ defined in $(3 \mathrm{a}, \mathrm{b})$. The first alternative (3a) describes, in the $a \rightarrow 0$ limit, the contact potential leading to non-trivial scattering of the so called renormalized $\delta(\mathbf{r})$ potential. This is the case discussed in the literature [10] that results in an energy dependent phase shift indicating the breaking of scale invariance, which has been identified by some authors as an anomaly in quantum mechanics [4]. On the other hand, when $v(a)=$ constant, the distribution defined by $(2,3 \mathrm{~b})$ has, in the $a \rightarrow 0$ limit, the same scaling behavior than the $\delta^{2}(\mathbf{r})$.

Outside the potential well, i.e. for $r>a$, we write the solution as:

$$
\psi_{\ell}^{e}(r, \theta)=\left(b_{\ell} J_{\ell}(k r)+c_{\ell} Y_{\ell}(k r)\right) e^{i \ell \theta} .
$$

Notice, for future reference, that comparing $(7,9)$ in the asymptotic region allow us to conclude $b_{\ell}=\cos \delta_{\ell}, c_{\ell}=-\sin \delta_{\ell}$.

On the other hand, in the internal region the solution is a linear combination of the modified $I$ and $K$ Bessel functions. Again the $K_{\nu}(\kappa r)$ function is discarded due to its singular behavior at the origin. Thus, for $r<a$ the solution is given by:

$$
\psi_{\ell}^{i}(r, \theta)=d_{\ell} I_{\ell}(\kappa r) e^{i \ell \theta},
$$

the coefficients $b_{\ell}, c_{\ell}$ entering in the external solution, for each value of the angular momentum $l$, can be expressed in terms of the $d_{\ell}$ coefficients of the internal solution by matching the wave functions and their derivatives at $r=a$, thus 


$$
\begin{aligned}
& d_{\ell}=\frac{1}{D}\left(\left(\frac{k}{\kappa}\right) J_{\ell}(k a) Y_{\ell}^{\prime}(k a)-J_{\ell}^{\prime}(k a) Y_{\ell}(k a)\right) b_{\ell}, \\
& c_{\ell}=\frac{1}{D}\left(J_{\ell}(k a) I_{\ell}^{\prime}(\kappa a)-\left(\frac{k}{\kappa}\right) J_{\ell}^{\prime}(k a) I_{\ell}(\kappa a)\right) b_{\ell},
\end{aligned}
$$

where

$$
D=\left(\frac{k}{\kappa}\right) Y_{\ell}^{\prime}(k a) I_{\ell}(\kappa a)-Y_{\ell}(k a) I_{\ell}^{\prime}(\kappa a)
$$

Let us first consider non-vanishing values of the angular momentum $l \neq 0$. Using the behavior of the Bessel functions for small arguments it is easily seen that both coefficients vanish in the $a \rightarrow 0$ limit. Thus, the phase shift and the scattering cross section vanish for non-zero values of the angular momentum, implying that the zero range potentials we are considering can only produce $\mathrm{S}$ wave scattering.

For zero angular momentum $l=0$, we consider two different cases. First we take for $v(a)$ the expression (3a) used in the literature [10]. In this case $c_{0}, d_{0}$ take the following non-zero values in the limit of vanishing radius $a \rightarrow 0$ :

$$
\begin{gathered}
d_{0}=-\frac{\ln \left(\frac{a}{a_{0}}\right)+\gamma}{\ln \left(\frac{k a_{0}}{2}\right)} b_{0}, \\
c_{0}=-\frac{\pi}{2} \frac{b_{0}}{\ln \left(\frac{k a_{0}}{2}\right)},
\end{gathered}
$$

from which we obtain the phase shift (see comment beneath eq. (9))

$$
\tan \delta_{0}(k)=-\frac{c_{0}}{b_{0}}=\frac{\pi}{2}\left(\ln \left(\frac{k a_{0}}{2}\right)\right)^{-1} .
$$

A second possibility we suggested in section two, is to consider $v(a)=v=$ constant. In this case the $c_{0}$ coefficient, and also the phase shift, vanish in the $a \rightarrow 0$ limit. The vanishing of the wave function at the origin in this case is also worth noticing.

¿From this exercise we obtain the following conclusions:

If the delta potential is treated in terms of the distribution $(2,3 \mathrm{a})$, which according to our reasoning breaks scale invariance at the classical level, then we get an energy dependent phase shift indicating the breaking of scale invariance in quantum mechanics.

If instead we use the distribution $(2,3 \mathrm{~b})$, then we get a vanishing phase shift, and scale invariance is an exact symmetry both at the classical and quantum level.

The triviality of the $S$ matrix is not related to the strength of the potential. This is concluded by comparing the behaviour at the origin of $(2,3 \mathrm{a})$ and $(2,3 \mathrm{~b})$. 


\section{SCATTERING WAVE FUNCTIONS.}

An alternative derivation of the results of the last section can be obtained in terms of the scattering wave functions. We start with the Schrödinguer equation and its formal solution written in terms of the Green function:

$$
\psi^{+}(\mathbf{r})=e^{i \vec{k} \cdot \mathbf{r}}-\int G\left(\mathbf{r}-\mathbf{r}^{\prime}\right) u\left(\mathbf{r}^{\prime}\right) \psi^{+}\left(\mathbf{r}^{\prime}\right) d^{2} \mathbf{r}^{\prime},
$$

where the Green function is defined by the differential equation:

$$
\left(\nabla^{2}+k^{2}\right) G\left(\mathbf{r}-\mathbf{r}^{\prime}\right)=-\delta\left(\mathbf{r}-\mathbf{r}^{\prime}\right)
$$

plus the condition that the wave function $\psi^{+}(\mathbf{r})$ describes a plane wave plus an outgoing "spherical" wave. For $\mathbf{r}-\mathbf{r}^{\prime} \neq 0$, the radial equation reduces to the Bessel equation (4), and the outgoing spherical condition selects $\mathcal{H}_{0}$ as the solution.

$$
G\left(\mathbf{r}-\mathbf{r}^{\prime}\right)=\frac{i}{4} \mathcal{H}_{0}\left(k\left|\mathbf{r}-\mathbf{r}^{\prime}\right|\right)
$$

The normalization $\left(c=-\frac{i}{4}\right)$ is fixed by the strength of the source at the origin

$$
c \int_{V \rightarrow 0} d^{2} r^{\prime}\left(\nabla^{2}+k^{2}\right) \mathcal{H}_{0}\left(k r^{\prime}\right)=-\int_{V \rightarrow 0} d^{2} r^{\prime} \delta\left(\vec{r}^{\prime}\right)=-1
$$

Returning to the scattering wave function, it is customary to show that asymptotically $\psi^{+}(\mathbf{r})$ describes a plane wave plus an outgoing "spherical" wave, defining in this way the scattering amplitude $f(\theta)$ :

$$
\psi^{+}(\vec{r})=e^{i \mathbf{k} \cdot \mathbf{r}}+f(\theta) \frac{e^{i\left(k r+\frac{\pi}{4}\right)}}{\sqrt{r}}
$$

For short range potentials [15] (i.e. if the potential $U(r)$ vanishes exactly for $r>a$ for some finite $a$ it is enough to approximate the argument of the Green function $G\left(\mathbf{r}-\mathbf{r}^{\prime}\right)$ by carrying aut an expansion in $\left(r^{\prime} / r\right)$ (remember that the vector $\mathbf{r}$ is understood to be directed towards the observation point at which the wave function is evaluated $(\mathbf{r} \rightarrow \infty)$ whereas the region that give rise to a nonvanishing contribution $\left(\mathbf{r}^{\prime}\right)$ is limited in space for a finite range potential) and considering the asymptotic expansion $\lim _{r \rightarrow \infty} \mathcal{H}_{0}\left(k r-k \frac{\mathbf{r} \cdot \overrightarrow{\mathbf{r}}^{\prime}}{r}\right)$. The same approximation is justified at length in Quantum Mechanics textbooks for the one dimensional case (to which our problem is reduced by the transformation $\varphi(r)=\tilde{\varphi}(r) / \sqrt{r}$ ) and for potentials falling off faster than $1 / r$. Thus, both for the $1 / r^{2}$ and $\delta^{2}(\mathbf{r})$ potentials, (12) is written as:

$$
\psi^{+}(\mathbf{r})=e^{i \mathbf{k} \cdot \mathbf{r}^{\prime}}+\frac{e^{-3 i \pi / 4}}{\sqrt{8 \pi k}} \frac{e^{i k r}}{\sqrt{r}} \mathcal{J}
$$

with

$$
\mathcal{J}=\int d^{2} r^{\prime} e^{-i k \frac{\mathbf{r} \cdot \mathbf{r}^{\prime}}{r}} u\left(r^{\prime}\right) \psi^{+}\left(\mathbf{r}^{\prime}\right) .
$$


Our aim is to obtain the phase shift. To achieve our goal we express the exact wave function $\psi(\mathbf{r})$ in terms of a complete set of functions $\varphi_{\ell}(\mathbf{r})$ :

$$
\psi^{+}(\vec{r})=\sum_{\ell=-\infty}^{\infty} N_{\ell} \varphi_{\ell}(r) e^{i \ell \theta}
$$

the $\varphi(r)$ functions are assumed to have the asymptotic behavior (in fact this behavior can be taken as definition of the phase shift $\delta_{\ell}$ )

$$
\left.\varphi_{\ell}(r)\right|_{r \rightarrow \infty}=\sqrt{\frac{2}{\pi k r}} \cos \left(k r-\frac{\ell \pi}{2}-\frac{\pi}{4}+\delta_{\ell}\right) .
$$

We also require the two dimensional version of the plane wave expansion in terms of "spherical" waves (states of definite angular momentum) [14]:

$$
e^{i k r \cos \theta}=\sum_{\ell=-\infty}^{\infty} i^{\ell} J_{\ell}(k r) e^{i \ell \theta} .
$$

The angular part of $\mathcal{J}$ can be explicitly calculated

$$
\mathcal{J}=2 \pi N_{\ell}(-i)^{\ell} e^{i \ell \theta} \int r^{\prime} d r^{\prime} J_{\ell}\left(k r^{\prime}\right) U\left(r^{\prime}\right) \varphi_{\ell}\left(r^{\prime}\right) .
$$

Furthermore, the asymptotic behavior in both sides of equation (14) leads to the relation $\left(x=k r-\frac{\ell \pi}{2}-\frac{\pi}{4}\right)$ :

$$
N_{\ell} \cos \left(x+\delta_{\ell}\right)=i^{\ell} \cos x-i\left(\frac{\pi}{2}\right) N_{\ell} \int_{0}^{\infty} r^{\prime} d r^{\prime} J_{\ell}\left(k r^{\prime}\right) U\left(r^{\prime}\right) \varphi_{\ell}\left(r^{\prime}\right) e^{i x} .
$$

Solving for each value of the angular momentum $l$ we obtain the coefficients $N_{\ell}$ and an integral expression for the phase shift (compare with of Roman [15]):

$$
\begin{gathered}
N_{\ell}=i^{\ell} e^{i \delta_{\ell}} \\
\sin \delta_{\ell}=-\frac{\pi}{2} \int_{0}^{\infty} r^{\prime} d r^{\prime} J_{\ell}\left(k r^{\prime}\right) U\left(r^{\prime}\right) \varphi_{\ell}\left(r^{\prime}\right) .
\end{gathered}
$$

Below we consider the $1 / r^{2}$ and $\delta(\mathbf{r})$ potentials separately.

\section{$1 / r^{2}$ potential}

For the $1 / r^{2}$ potential the exact solution is $\varphi_{\ell}(r)=J_{\ell}(k r)$. Substituting this in (15), the phase shift takes the form:

$$
\sin \delta_{\ell}=-m \pi \lambda \int_{0}^{\infty} r d r J_{\ell}(k r) \frac{1}{r^{2}} J_{\nu(\ell)}(k r) .
$$

This integral is tabulated in [14] 


$$
\int_{0}^{\infty} J_{\alpha}(x) J_{\beta}(x) x^{-\gamma} d x=\frac{\Gamma(\lambda) \Gamma\left(\frac{1}{2}(\nu+\mu-\lambda+1)\right)}{2^{\lambda} \Gamma\left(\frac{1}{2}(-\nu+\mu+\lambda+1)\right) \Gamma\left(\frac{1}{2}(\nu+\mu+\lambda+1)\right) \Gamma\left(\frac{1}{2}(\nu-\mu+\lambda+1)\right)}
$$

using the reflection formula $\Gamma(z) \Gamma(1-z)=\pi \csc (\pi z)$ we finally we obtain:

$$
\delta_{\ell}=\frac{\pi}{2}(\ell-\nu(\ell))+n \pi, \quad n=0,1, \ldots
$$

the phase shift coincides with relation (8) obtained through the asymptotic behavior of the exact eigenfunctions. For completeness we quote the scattering wave function:

$$
\psi^{+}(\mathbf{r})=\sum_{\ell=-\infty}^{\infty} e^{i \delta_{\ell}} i^{\ell} J_{\nu(\ell)}(k r) e^{i \ell \theta}
$$

Comparing (16) and (13) we obtain the scattering amplitude:

$$
\left.f(\theta)=\frac{1}{\sqrt{2 \pi k}} \sum_{\ell=-\infty}^{\infty}\left(e^{2 i \delta \ell}-1\right) e^{i \ell \theta}\right) .
$$

It can be observed that, as scale invariance requires, the differential cross section is also energy independent, that is the angular probability of scattering and the full cross section are not affected by the energy of the incoming particle.

\section{$\delta^{2}(\mathbf{r})$ potential.}

Our task is to analyze the behavior of the integral representation of the phase shift. To this end we notice that, independently of the distribution we use to regularize the delta potential, the integral is over the finite interval $(0, a)$ and the exact solution $\psi$ is given by (9), therefore:

$$
\sin \delta_{\ell}=-\frac{\pi}{2} \lim _{a \rightarrow 0} \int_{0}^{\infty} r^{\prime} d r^{\prime} J_{0}\left(k r^{\prime}\right) \frac{v(a)}{\pi a^{2}} d_{0} I_{0}\left(\kappa r^{\prime}\right)
$$

For non vanishing values of the angular momentum, and in the $a \rightarrow 0$ limit, the integral appearing in (17) is finite whereas in the same limit $v(a) \cdot d_{0}=0$. Thus, as in the previous section, we conclude the absence of scattering for contact potential and $l \neq 0$.

For the $\mathrm{S}$ wave $(l=0)$ we first consider $v(a)$ given by Eq. (3a). Using $(10 \mathrm{~b})$ the phase shift reduces to:

$$
\sin \delta_{0}=\frac{\pi}{2} \frac{b_{0}}{\ln \left(\frac{k a_{0}}{2}\right)}
$$

which, recalling the comment beneath Eq. (9), can be re-written as:

$$
\tan \delta_{0}=\frac{\pi}{2} \frac{1}{\ln \left(\frac{k a_{0}}{2}\right)}
$$


the energy dependence of this phase shift indicates the breaking of scale invariance, in agreement with (11), with previous analysis in the literature [10] and reproduced in the previous section. It should be clear by now that a different result is obtained if instead of (3a) we consider $v(a)=v=$ constant. Indeed, in this case, in the $a \rightarrow 0$ limit we obtain a vanishing phase shift.

\section{SELF ADJOINT EXTENSION}

In his contribution to Beg's Memorial Volume, Jackiw proved that the self adjoint extension is an alternative approach to the description of the two dimensional renormalized delta potential [9]. As emphasized by Jackiw, this approach has the advantage - besides providing a more satisfactory mathematical frame - that it avoids the need to deal with infinite quantities, and allow a clear understanding of why the symmetry is broken quantum mechanically. On the other hand, in the previous section we argued that the conventional approach to the regularized delta potential breaks scale invariance whereas that a properly regularized delta potential preserves the symmetry both at the classical and quantum level and leads to a trivial S matrix. It is our purpose in this section to show that the triviality of the $\mathrm{S}$ matrix is also consistent with the self adjoint extension approach. We are not claiming that the self-adjoint extension treatment is not valid or incorrect, we only remark that, under different assumptions, the results of [9] admit a different interpretation.

Consider the radial equation (4). Extracting a $\sqrt{r}$ factor from the wave function (i.e. instead of $\varphi$ it is convenient to introduce the function $\tilde{\varphi}(r)=\sqrt{r} \varphi(r)$ ) this problem is reduced to a one dimensional quantum mechanical system restricted to the half line:

$$
\frac{d^{2} \tilde{\varphi}}{d r^{2}}-\frac{\left(\ell^{2}-\frac{1}{4}\right)}{r^{2}} \tilde{\varphi}-2 U(r) \tilde{\varphi}+k^{2} \tilde{\varphi}=0
$$

We begin by considering the self adjoint condition for a free particle (notice that this condition is not modified by adding an hermitean potential),

$$
\int_{0}^{\infty} \varphi_{1}^{*} \frac{d^{2} \tilde{\varphi}_{2}}{d r^{2}} d r=\int_{0}^{\infty}\left(\frac{d^{2} \tilde{\varphi}_{1}}{d r^{2}}\right)^{*} \tilde{\varphi}_{2} d r
$$

integrating by parts and assuming that $\varphi(r)$ vanishes at infinity (to assure normalizability), it follows that the hamiltonian is self-adjoint on the set of wave function that satisfies the boundary condition:

$$
\lim _{r \rightarrow 0}\left(\varphi_{1}^{*} \frac{d \tilde{\varphi}_{2}}{d r}-\left(\frac{d \tilde{\varphi}_{1}}{d r}\right)^{*} \varphi_{2}\right)=0
$$

Thus the self-adjoint property is not limited to the operator (the hamiltonian in this case) but includes also the space of wave functions. When the $r \rightarrow 0$ limit exist, both for the function and its derivative, (19) is conveniently summarized in the condition [16]:

$$
\tilde{\varphi}^{\prime}(0)=-c \tilde{\varphi}(0)
$$


in the nomenclature of mathematical physics $[9,17]$ we say that the free hamiltonian admits a one parameter family of self adjoint extensions labeled by the real parameter $c$. The physical interpretation of these boundary conditions is as follows. The function $e^{-i k r}\left(e^{i k r}\right)$ is a plane wave moving to the left (right) with momentum $k>0$, i.e. is an outgoing (incoming) wave of momentum k. Clearly these are not square integrable functions; however we ignore that since we are only interested in its behavior near the origin. Neither $e^{-i k r}$ nor $e^{i k r}$ belong to the space of functions leading to a self-adjoint free hamiltonian. At this point it is convenient to introduce the wave function:

$$
\chi(r)=e^{-i k r}+\alpha e^{i k r}
$$

If $\alpha=\frac{i k-c}{i k+c}$, then $\chi(r)$ satisfies the boundary condition (20). Thus the free hamiltonian together with the boundary condition (20) generate the dynamics in which a plane wave of momentum $k$ is scattered. Different self-adjoint extensions (different $c^{6} s$ ) produce different $\left(\alpha^{6} s\right)$, i.e. different self adjoint extensions correspond to different physics (potentials).

The self-adjoint extension method can be used in different ways, in particular it can be used to test the symmetry after quantization. Indeed if $\psi(r)$ is a wave function that satisfies the boundary condition (20), if $\mathbf{D}$ is the generator of a symmetry transformation, and if the symmetry is not broken by the quantization process, then $\psi^{\prime}(r)=\mathbf{D} \psi(r)$ must also satisfy the boundary condition (20). Below we apply this criteria to the $\delta^{2}(\mathbf{r})$ potential.

Following Jackiw [9], to apply the self adjoint method we consider the exact, external S wave $(l=0)$ wave function which for the $\delta^{2}(\mathbf{r})$ potential have the following behavior at the origin (recall that $\tilde{\varphi}(r)=\sqrt{r} \varphi(r)$ :

$$
\begin{gathered}
\tilde{\varphi}_{0}(r)=b_{0} \sqrt{r}\left(J_{0}(k r)+\frac{c_{0}}{b_{0}} Y_{0}(k r)\right) \\
\rightarrow\left[\sqrt{r}\left(1+\frac{2 \gamma}{\pi} \tan \delta_{0}\right)+\frac{2 \tan \delta_{0}}{\pi} \sqrt{r} \log \left(\frac{k r}{2}\right)\right] \underset{r \rightarrow 0}{\rightarrow} 0 .
\end{gathered}
$$

Eventhough the wave function vanishes at the origin, since the derivative $\tilde{\varphi}^{\prime}(r)$ is singular at that point:

$$
\tilde{\varphi}_{0}^{\prime}(r) \underset{r \rightarrow 0}{2 \sqrt{r}}\left(1+\frac{4 \tan \delta_{0}}{\pi}\left(1+\frac{\gamma}{2}\right)\right)+\frac{1}{\sqrt{r}} \frac{\tan \delta_{0}}{\pi} \log \left(\frac{k r}{2}\right)
$$

then, for consistency, we consider condition (19) instead of (20). For $\tilde{\varphi}_{1}$ and $\tilde{\varphi}_{2}$ we take free particle solutions $\varphi_{0}^{i}=b_{0}^{i}\left(J_{0}(k r)+\tan \delta_{0}^{i} Y_{0}(k r)\right)$ where $\delta_{0}^{i}$ stands for the phase shift of the $i$ - esim solution (see comment beneath eq. (9)). It is straightforward to show that the selfadjointedness condition (19) requires $\delta_{0}^{1}=\delta_{0}^{2}$, i.e. that the hamiltonian is selfadjoint on the class of functions with the same phase shift. This characteristics can be used to test the symmetry after quantization. If the quantization process preserves the symmetry, then the 
symmetry generator must not change the ratio of the $J_{0}, Y_{0}$ contributions in $(22)$. Given $\mathbf{D}$ $H t+\frac{i}{2}(r \partial r+1)$, we see that only the $r \partial r$ term can change the ratio under consideration. Applying this criterium to (22), we conclude that scale invariance survives the quantization process only for $\delta=0$. Thus, the self adjoint extension approach is consistent with the result obtained by explicit evaluation of the phase shift (see also [9]).

\section{SUMMARY}

In this paper we considered the quantum mechanics of scale invariant potentials $\left(1 / r^{2}\right.$ and $\delta^{2}(\mathbf{r})$ potentials). We have shown that a scale invariant regularization of the $\delta^{2}(\mathbf{r})$ potential leads to a trivial $\mathrm{S}$ matrix. The triviality of the $\mathrm{S}$ matrix is not related to the strength of the potential, in fact the scale invariant regularization has a stronger singularity at the origin that the regularization leading to a non trivial $\mathrm{S}$ matrix. We conclude that scale invariance survives the process of quantization and that the symmetry breaking of scale invariance reported in the literature for the $\delta^{2}(\mathbf{r})$ potential is an example of explicit breaking and not an anomaly. We have indicated how the same result can be consistently obtained within the self-adjoint extension approach.

One is tempted to extrapolate our conclusion to quantum field theory. At that level the question is wheter the renormalization is part of the quantization or not; if it is not, then dimensional transmutation could be considered an explicit breaking and not an anomaly!!! 


\section{REFERENCES}

1.- L.D. Landau, E.M., Lifshitz "Mechanics". Ed. Riverte (1978).

2.- J.M. Levy Leblond, Am. J. Phys. (1971) 502.

3.- See for example J. Govaerts "Hamiltonian Quantisation and Constrained Dynamics". Leuven University Press (1991).

4.- B. Holstein Am. J. Phys. 61 (1993) 142.

5.- G.V. Dunne, R. Jackiw and C.A. Trugenberger, Phys. Rev. D41 (1990) 661.

6.- A. Cabo and J.L. Lucio M. Phys. Lett. A 219, (1996) 155.

7.- B. Holstein, Am. J. Phys. 61 (1993) 142, C. Manuel R. and Tarrach. Phys. Letts. B 328 (1994) 113. See also ref. 9.

8.- M.A.B. Beg and R. Furlong, Phys. Rev. D31, 1370 (1985).

9.- R. Jackiw, in M.A.B. Beg Memorial Volume, eds. A. Ali and P. Hoodbhoy (World Scientific, Singapore, 1991).

10.- P. Godzinsky and R. Tarrach. Am. J. Phys. 59 (1991) 70, see also L.R. Mead and J. Godines, Am. J. Phys. 59, (1991) 935, C. Manuel and R. Tarrach, Phys. Letts. B 328, (1994) 113.

11.- S.K. Adhikari and T. Frederico. Phys. Rev. Letts. 74, (1995) 4572

12.- See first paper of ref. 10 and references therein.

13.- A. Cabo, J.L. Lucio M. and M. Napsuciale. Ann. Phys. 244 (1995) 1 and references therein.

14.- M. Abramowitz and I. Stegun, Handbook of Mathematical Functions Dover, New York (1970).

15.- P. Roman. "Advanced Quantum Theory". Adison Wesley, Massachussetts (1965).

16.- E. D’Hoker and L. Vinet Commun. Math. Phys. 97 (1985) 391.

17.- Reed M., Simon B.: Fourier Analysis and Selfadjointedness New York, Academic Press (1975). 